\title{
miRNA expression profiling identifies DSPP regulators in cultured dental pulp cells
}

\author{
XIN HUANG, SHUJUN XU, JIE GAO, FEI LIU, JING YUE, TING CHEN and BULING WU \\ Department of Stomatology, Nanfang Hospital, Southern Medical University, College \\ of Stomatology, Southern Medical University, Guangzhou, P.R. China
}

Received April 8, 2011; Accepted May 24, 2011

DOI: $10.3892 / \mathrm{ijmm} .2011 .721$

\begin{abstract}
Dentin sialophosphoprotein (DSPP), an important marker of odontoblast differentiation, is a prerequisite for tooth development and mineralization; however, the molecular mechanisms of both temporal and spatial regulation remain unknown. MicroRNAs (miRNAs) provide an additional level of control beyond that of transcription factors, which regulate post-transcriptional control of gene expression. The present study was designed to provide a first attempt at an in-depth analysis of dental pulp cells at various odontoblastic differentiation stages to obtain miRNA differential expression patterns, and to determine the contribution of miRNAs in the expression of DSPP during odontoblast differentiation. Dual luciferase reporter assays and qRT-PCR were used to validate miRNAs identified from bioinformatic analyses to determine whether they were able to regulate the DSPP gene in dental pulp cells cultured in a mineralizing medium. The results presented here suggest that DSPP is regulated post-transcriptionally by mir32, mir885-5p and mir586 during odontoblast differentiation.
\end{abstract}

\section{Introduction}

Dentinogenesis is a process of continuous matrix deposition that occurs throughout the life of a tooth. Odontoblasts, a monolayer of cells located at the periphery of the pulp, secrete the unmineralized dentin matrix (predentin), and are responsible for the mineralization of this matrix (dentin). Odontoblast cytodifferentiation results in the expression of tissue-specific gene products that form the structure of the dentin extracellular matrix. Dentin sialophosphoprotein (DSPP), which is thought to be tooth-specific $(1,2)$, was first cloned from developing teeth and later found to be expressed in bone (3), neoplasms [e.g., breast, lung (4), and prostate tumors (5)], and in soft tissues

Correspondence to: Dr BuLing Wu, Department of Stomatology, Nanfang Hospital, Southern Medical University, College of Stomatology, Southern Medical University, Guangzhou, P.R. China E-mail: bulingwu@yahoo.cn

Key words: dental pulp cells, dentin sialophosphoprotein, microRNA, dual luciferase reporter assay including the kidney (6) and salivary glands (7). However, DSPP both inside and outside of the dentin is similar in nature but different in quantity, with the amount of DSPP in bone about $1 / 400$ of that found in dentin (3). Therefore, the relative expression of the DSPP protein and gene expression can be used as a marker for odontoblasts.

Specifically, DSPP is expressed transiently by presecretory ameloblasts, as well as long-term by odontoblasts throughout dentinogenesis $(2,8)$. The primary DSPP transcript is processed into two major polypeptides, recognized as dentin sialoprotein (DSP) and dentin phosphoprotein (DPP) (1). Extensive evidence indicates that DSPP plays a crucial role during both enamel and dentin biomineralization and/or the formation of the structural diversity of tooth layers (i.e., enamel, mantle dentin, predentin and orthodentin) (1,9,10). DSPP mutations are reported to be associated with DD-II, DGI-II (11-20) and DGI-III (21), resulting in abnormal dentin formation. Furthermore, DSPP knockout mice display a widened predentin zone and develop defective mineralization similar to human dentinogenesis imperfecta III (22).

Understanding the processes that regulate the tissuespecific gene expression is of importance in developmental biology. In particular, the coordinated development of odontoblast cells depends on the adjustment of various regulators that are responsible for the induction of cell differentiation and the expression of tooth-specific genes. Previous studies have shown that dentinogenesis, a complex process in which multiple signaling pathways induce dentin formation, is controlled by many growth and transcription factors (23-33). However, the regulation of DSPP is not fully understood, particularly the understanding of its post-transcriptional regulation.

MicroRNAs (miRNAs) provide an additional level of control beyond that of transcription factors. miRNAs are a class of small noncoding RNA molecules that regulate gene expression post-transcriptionally in eukaryotic cells (34-39). miRNAs are 22 nucleotide (nt) single-stranded RNAs that silence gene expression by binding to target mRNAs. Mature miRNAs target the 3' untranslated regions (3'UTR) of genes by complementary base-pair binding. Furthermore, mature miRNAs can alter the expression of genes by binding to the coding regions as well as the $5^{\prime}$ untranslated regions (5'UTR) $(40,41)$.

To understand the molecular mechanisms regulating dentinogenesis, we utilized computer analysis of the 3'UTR 
Table I. Oligonucleotides used in this study.

\begin{tabular}{ll}
\hline hsa-mir32 RT & 5'-CTCAACTGGTGTCGTGGAGTCGGCAATTCAGTTGAGTGCAACTT-3' \\
hsa-mir855-5p RT & 5'-CTCAACTGGTGTCGTGGAGTCGGCAATTCAGTTGAGAGAGGCAG-3' \\
hsa-mir586 RT & 5'-CTCAACTGGTGTCGTGGAGTCGGCAATTCAGTTGAGGGACCTAA-3' \\
hsa-mir32 F & 5'-ACACTCCAGCTGGGTATTGCACATTACTAAGT-3' \\
hsa-mir885-5p F & 5'-ACACTCCAGCTGGGTCCATTACACTACCCTGCCT-3' \\
hsa-mir586 F & 5'-ACACTCCAGCTGGGTATGCATTGTATTTTTAGGT-3' \\
miRNA_R & 5'-CTCAACTGGTGTCGTGGA-3' \\
U6-F & 5'-CTCGCTTCGGCAGCACA-3' \\
U6-R & 5'-AACGCTTCACGAATTTGCGT-3'
\end{tabular}

${ }^{a}$ miRNA_R represents the universal reverse primer in conjunction with a sequence-specific forward primer for hsa-mir32, hsa-mir885-5p and hsa-mir586.

and identified several potential regulatory miRNAs. We then performed a dual luciferase reporter assay using RNA extracts isolated from human dental pulp cells to determine whether miRNAs play a role in the regulation of the DSPP gene.

\section{Materials and methods}

Cell culture. Primary human dental pulp cells were isolated from explanted healthy pulp of impacted third molars, following an established protocol $(42,43)$. The cells were cultured in a growth medium containing Dulbecco's modified Eagle's medium (Gibco) with $10 \%$ fetal bovine serum, $1 \%$ L-glutamine, $10,000 \mathrm{IU} / \mathrm{ml}$ penicillin $\mathrm{G}, 100,000 \mathrm{mg} / \mathrm{ml}$ streptomycin sulfate, and $25 \mathrm{mg} / \mathrm{ml}$ amphotericin $\mathrm{B}$ at $37^{\circ} \mathrm{C}$, $5 \% \mathrm{CO}_{2}$ condition. Cells from the second passages were used for all experiments. To activate differentiation and mineralization, DPCs were cultured in a mineralizing medium containing growth medium, $50 \mathrm{mg} / \mathrm{ml}$ ascorbic acid, $10 \mathrm{mM}$ $\beta$-glycerophosphate, and $0.01 \mathrm{mM}$ dexamethasone (Sigma).

T293 cells were grown in Dulbecco's modified Eagle's medium (Gibco) supplemented with $10 \%$ fetal bovine serum.

Mineralization staining. Mineralization of cultured DPCs was determined using Alizarin Red (AR) staining. After Day 14, the cell layer was washed with PBS and fixed in 10\% formaldehyde (Sigma-Aldrich) at room temperature for $15 \mathrm{~min}$, then washed in duplicate with excess $\mathrm{dH}_{2} \mathrm{O}$ prior to the addition of $1 \mathrm{ml}$ of $40 \mathrm{mM}$ AR ( $\mathrm{pH} 4.1$ ). The plates were incubated at room temperature for 20 min under gentle shaking. Following aspiration of the unincorporated dye, the plates were washed twice with $\mathrm{dH}_{2} \mathrm{O}$ and visualized using phase microscopy (Nikon).

Reverse transcription-PCR. Total-RNA was prepared from mineralized cultured dental pulp cells from Days 7 and 14 . First-strand cDNA synthesis and PCR processes were performed per the manufacturer's instructions. The primer sets for DMP-1 were sense, 5'-TGGGTTTGTTGTGATAGG-3'; antisense, 5'-GGAAGAGGTGGTGAGTGA-3'. The reactions were incubated at $94^{\circ} \mathrm{C}$ for $5 \mathrm{~min}$ for one cycle and then $94^{\circ} \mathrm{C} / 45 \mathrm{sec}, 51^{\circ} \mathrm{C} / 45 \mathrm{sec}, 72^{\circ} \mathrm{C} / 1 \mathrm{~min}$ for 40 cycles, with a final 10 -min extension at $72^{\circ} \mathrm{C}$. The primer for DSPP was sense, 5'-GGCAGTGACTCAAAAGGAGC-3'; antisense, 5'-TGCTG
TCACTGTCACTGCTG-3'. The reactions of DSPP were incubated at $94^{\circ} \mathrm{C}$ for $5 \mathrm{~min}$ for one cycle and then $94^{\circ} \mathrm{C} / 45 \mathrm{sec}$, $54^{\circ} \mathrm{C} / 45 \mathrm{sec}, 72^{\circ} \mathrm{C} / 1 \mathrm{~min}$ for 35 cycles, with a final $10-\mathrm{min}$ extension at $72^{\circ} \mathrm{C}$. Following amplification, a 10 min extension was performed with 72 reactions of DSPP using $1.5 \%$ agarose gel electrophoresis and visualized using ethidium bromide staining.

Bioinformatic analyses. Bioinformatic scans of the 3'UTR of DSPP were conducted using four web-based miRNA target prediction programs: miRGen-miRanda, mirBase, microRNA and TargetScan 3.0 (44-47). miRNAs were chosen based upon their targeted prediction by more than one program, conservation of the binding region, and strength of the predicted interaction.

Dual luciferase reporter assay. The psiCHECK2 luciferase vector (Promega) was used for the dual luciferase assays. The 3'UTR of DSPP was inserted using the XhoI/NotI sites. Fragments of the 3'UTR of DSPP were obtained by PCR using DSPP cDNA as the template. The primers for DSPP 3'UTR were XhoIF, 5'-CCGCTCGAGAACAAAAGAAAAACCCGTAAG-3', NotIR, 5'-ATAAGAATGCGGCCGCTTCTGTGTGAAGTAT TAAAGAAT-3'. PCR products were digested using XhoI/NotI and cloned into the psiCHECK2 plasmid (Promega).

Luciferase assays were performed using the DualLuciferase assay kit as described (Promega). 293-T cells were co-transfected in 24 -well plates with the indicated psiCHECK2 luciferase construct $(0.5 \mu \mathrm{g} /$ well $)$ and miRNA $(20 \mu \mathrm{M})$ using Lipofectamine 2000 . Following $48 \mathrm{~h}$, the cells were harvested in passive lysis buffer, and luciferase activity was determined using a GloMax ${ }^{\mathrm{TM}}$ 20/20 luminometer (Promega). The luciferase data are expressed as a ratio of Renilla luciferase (RL) to firefly luciferase (FL) to normalize for transfection variability between samples. Luciferase experiments were repeated in triplicate using independent samples, as indicated.

$q R T-P C R$. To validate the above data, expression of the three miRNA genes was determined using qRT-PCR analysis. Dental pulp cells cultured in a mineralizing medium were able to differentiate into odontoblast-like cells. Samples were 

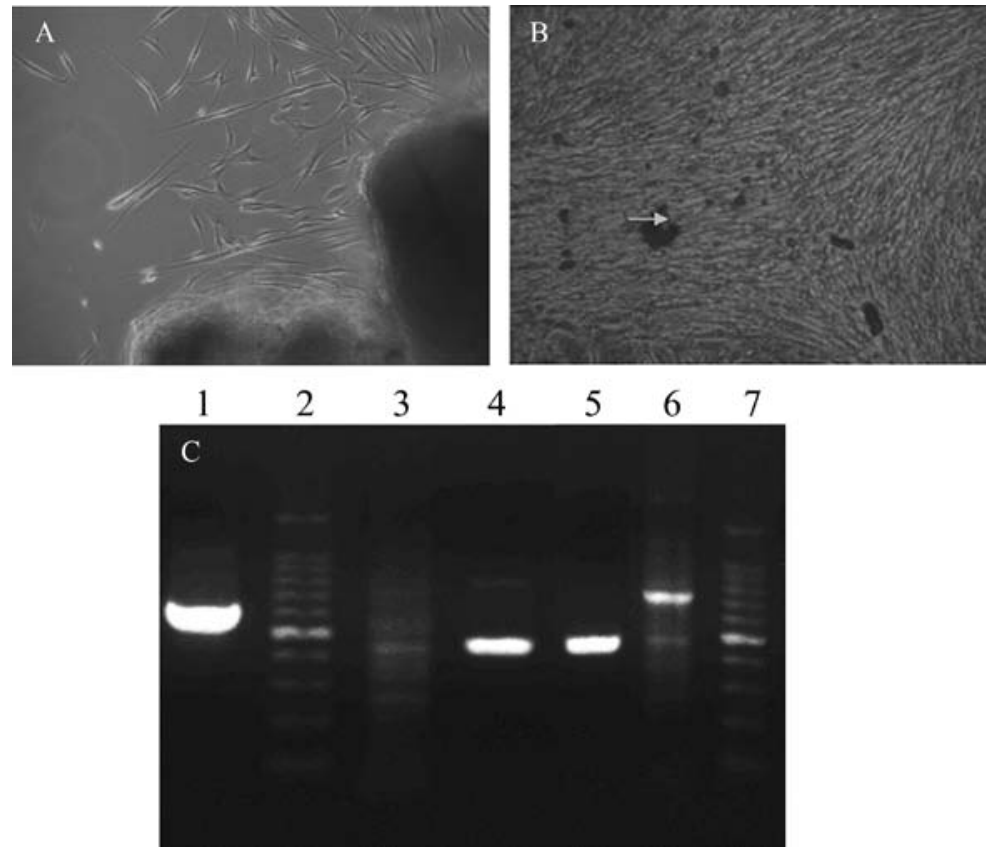

Figure 1. Cultivation and characteristics of dental pulp cells. (A) Dental pulp cells were dispersed from the tissue after about 14 days. Confluent cultures were collected using trypsinization ( $0.2 \%$ trypsin and $0.02 \%$ EDTA) and subcultured. (B) Cells from second passages were used for the mineralization culture with Alizarin Red staining. After about 10 days, mineralized nodules (arrow) formed and became more condensed. (C) RT-PCR for the marker gene DSPP and DMP1, respectively, on Days 14 and 7, were strongly expressed (lane 1, $\beta$-actin; lane 2, 100 bp DNA ladder marker; lane 3, DSPP on Day 7, not expressed; lane 4, DMP1 on Day 7; lane 5, DMP1 on Day 14; lane 6, DSPP on Day 14; lane 7, 100 bp DNA ladder marker).

harvested for the isolation of RNA at 7, 10, 14, 21 or 28 days of differentiation to detect quantitative changes in gene DSPP and three miRNAs. Cells were cultured in a growth medium that served as the control.

RNA was isolated from cells using TRIzol (Invitrogen) as recommended by the manufacturer. For quantitative RT-PCR analysis, $0.1 \mu \mathrm{g}$ of RNA per reaction was used with the Quantitech SYBR-Green RT-PCR kit and primers specific for DSPP. To quantify miRNA expression, total-RNA was reverse transcribed for use in two-step quantitative RT-PCR using the stem-loop method (48-50). The resulting cDNA was subjected to real-time qRT-PCR using the universal reverse primer in conjunction with a sequence-specific forward primer for hsa-mir32, hsa-mir885-5p and hsa-mir586. Each sample was performed in triplicate, and the results were normalized using primers to 18S rRNA (for DSPP) or U6 (for miRNA analysis) (Table I). Results are expressed as fold change in expression relative to the control sample calculated using the equation $\mathrm{RQ}=2^{-\Delta \Delta \mathrm{Ct}}$.

Statistical analyses. All data are presented as the mean \pm SD $(n \geq 3)$. We performed statistical analysis using ANOVA, and $\mathrm{P}<0.05$ was considered statistically significant.

\section{Results}

Characteristics of dental pulp cells. It has been demonstrated that dexamethasone (Dex) and/or $\beta$-glycerophosphate $(\beta-\mathrm{GP})$ in the culture medium of dental pulp cells can induce odontoblast features (51-60). Cultured dental pulp cells were spindle-shaped in appearance with extended cytoplasmic processes and were grown to a near-confluent state. Following 10 days, mineralized nodules formed and became more condensed. Alizarin Red staining of mineralized nodules in representative cell cultures is demonstrated in Fig. 1B. Our results suggest that expression of matrix protein-1 (DMP-1) is detected from Day 7 and DSPP from Day 14, which are two dentin-specific markers (Fig. 1C). Previous studies and biochemical confirmation of the differentiated DPC phenotypes confirm DPCs as a powerful model for our study.

Bioinformatic analyses for identification of miRNAs that regulate DSPP. Based on the stem-loop feature of the miRNA and cross-species comparison, a number of computational algorithms have been developed to predict miRNAs from the genome. Bioinformatic scans are the in silico standard for assembling a list of candidate miRNAs predicted to target the 3'UTR of a given RNA. We used four programs (TargetScan, mirBase, microRNA and miRGen-miRanda) to generate a list of potential miRNAs with a high probability of binding to the 3'UTR of DSPP (Table II). To narrow these lists and increase our probability of identifying miRNAs capable of regulating DSPP post-transcriptionally, we chose to further examine ten miRNAs that were identified by at least two search algorithms (Table III).

miRNAs regulate DSPP by dual luciferase reporter assay. To test whether the 3'UTR of DSPP contained sequences capable of interacting with potential miRNAs, luciferase reporter assays were performed. The 3'UTR of DSPP was inserted into the psiCHECK ${ }^{\mathrm{TM}}-2$ Vector using the XhoI/NotI sites and replicated with a vector. The relevant DSPP 3'UTR fragment was fused to luciferase and transiently transfected into 293-T cells, then analyzed for its ability to repress luciferase activity. When adding a miRNA that is able to interact with DSPP 3'UTR, the bioluminescence reaction of Photinus pyralis 
Table II. Potential miRNAs targeting the DSPP 3'UTR.

\begin{tabular}{llll}
\hline TargetScan & \multicolumn{1}{c}{ mirBase } & microRNA.org & miRGen-miRanda \\
\hline hsa-mir513p & hsa-mir593 & hsa-mir513p & hsa-mir205 \\
hsa-mir885-5p & hsa-mir221 & hsa-mir885-5p & hsa-mir221 \\
hsa-mir337-3p & hsa-mir32 & hsa-mir361-3p & hsa-mir451 \\
hsa-mir384 & hsa-mir506 & hsa-mir452 & hsa-mir506 \\
hsa-mir384-3p & hsa-mir646 & hsa-mir659 & hsa-mir519d \\
hsa-mir578 & hsa-mir512-5p & hsa-mir586 & hsa-mir119e \\
hsa-mir432 & hsa-mir587 & hsa-mir593 & hsa-mir520e \\
hsa-mir361-3p & hsa-mir101 & hsa-mir1205 & hsa-mir520h \\
hsa-mir452.h & hsa-mir571 & hsa-mir548 & hsa-mir577 \\
hsa-mir659 & hsa-mir540-5p & hsa-mir186 & hsa-mir32 \\
hsa-mir1205 & hsa-mir346 & & \\
hsa-mir593 & hsa-mir217 & & \\
hsa-mir1286 & hsa-mir183 & & \\
hsa-mir1184 & hsa-mir31 & & \\
hsa-mir586 & hsa-mir489 & & \\
hsa-mir495 & & & \\
hsa-mir1192 & & & \\
\hline
\end{tabular}

Four miRNA target search algorithms (TargetScan, mirBase, microRNA.org, miRGen-miRanda) were utilized to generate a list of potential miRNA binding sites within the DSPP 3'UTR.

Table III. The ten miRNAs chosen for further study.

miRNA prediction programs

\begin{tabular}{lcccc}
\cline { 2 - 5 } miRNA & mirBase & miRGen-miRanda & TargetScan & microRNA.org \\
\hline hsa-mir513p & - & - & + & + \\
hsa-mir885-5p & - & - & + & + \\
hsa-mir361-3p & - & - & + & + \\
hsa-mir659 & - & - & + & + \\
hsa-mir1205 & - & - & + & + \\
hsa-mir586 & - & - & + & + \\
hsa-mir593 & + & - & - & - \\
hsa-mir221 & + & + & - & - \\
hsa-mir32 & + & + & - & - \\
hsa-mir506 & + & + & + \\
\hline
\end{tabular}

There are six miRNAs predicted by the biology software TargetScan and microRNA.org, and three by mirBase and miRGen-miRanda. An additional miRNA was predicted by three of the software, and is considered a potentially important regulator of DSPP 3'UTR.

luciferase was annihilated because miRNA inhibits translation of vector mRNA. This construct allowed us to quickly and quantitatively evaluate miRNA effects on the 3'UTR of DSPP. The DSPP 3'UTR fragment inserted into the psiCHECK2 luciferase vector is shown in Fig. 2. The relative luciferase activity in 293-T cells transfected with the luciferase vector alone was arbitrarily set at $100 \%$ for comparison. The binding ability of the ten predicted miRNAs with DSPP is shown in Fig. 3. mir885-5p, mir586 and mir32 (Fig. 4) all significantly $\left({ }^{* *} \mathrm{P}<0.01,{ }^{*} \mathrm{P}<0.05\right)$ reduced luciferase activity when compared to the negative scrambled miRNA and the luciferase vector alone, while mir513P, mir361-3P, mir659, mir1205 and mir506 (Fig. 5) did not. mir593 and mir221 (Fig. 6) significantly repressed luciferase activity in comparison to the negative scrambled miRNA ( $\mathrm{P}<0.05)$, but was not different with the cells transfected with the luciferase vector alone, suggesting the probability of repressed luciferase activity. 


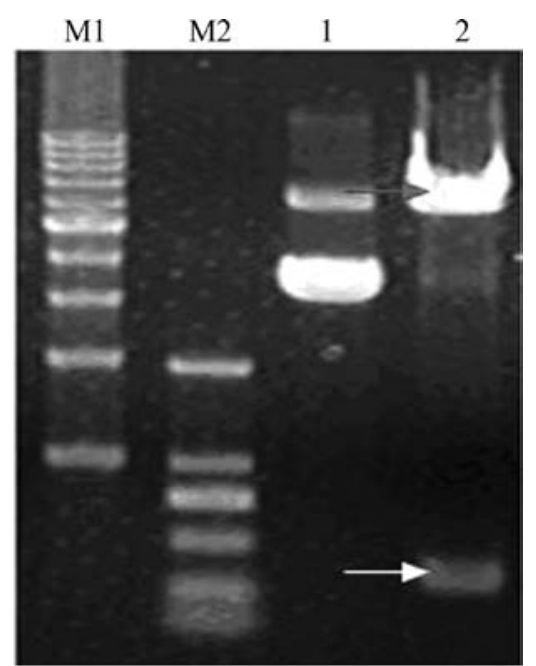

Figure 2. A DSPP 3'UTR fragment was inserted into the psiCHECK-2 vector (lane 2). The arrow indicates the DSPP $3^{\prime}$ UTR fragment (305 bp, below) and the vector fragment (7 kb, above) after digestion of psiDSPP by Xhol/NotI. (lane 1, psiDSPP recombinant plasmid).

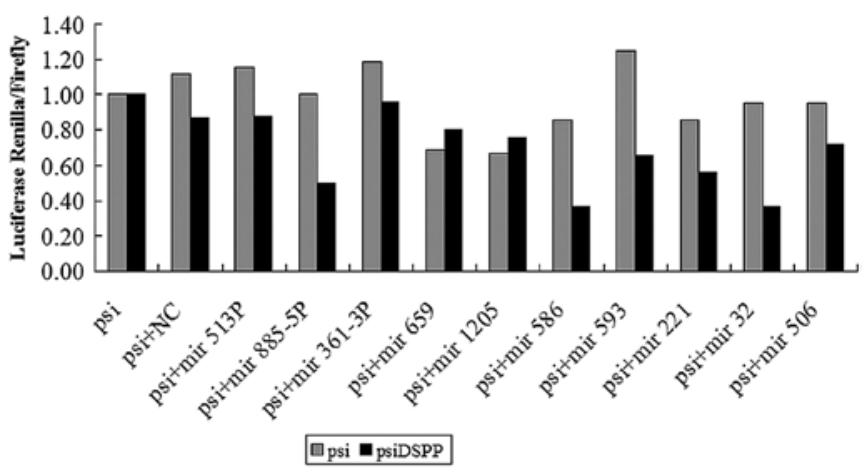

Figure 3. The binding ability of the predicted DSPP-targeting miRNAs. Luciferase assays were performed following co-transfection of the DSPP 3'UTR luciferase reporter and the indicated miRNA-precursors. A luciferase signal is shown after Renilla luciferase readings were normalized to firefly luciferase. Psi represents the luciferase vector alone. PsiDSPP represents the luciferase vector with a DSPP 3'UTR fragment. NC does not code for any known miRNA.

Identification of miRNAs that regulate DSPP by $q R T-P C R$. qRT-PCR analysis with commercial primer sets for reverse transcription of specific mature miRNAs can be used for amplification and detection of cDNA products using SYBRGreen fluorescence. To validate the above data, the expression of three miRNA genes in the process of dental pulp cell differentiation was determined using qRT-PCR analysis.

The DSPP transcript was predominantly expressed in odontoblasts and transiently in preameloblasts along with an involvement with odontoblast differentiation and mineralization, which is used as an indicator of odontoblastic differentiation $(2,61)$. Dental pulp cells cultured in mineralizing medium exhibited odontoblastic features, including increasing DSPP; undifferentiated dental pulp cells also exhibit DSPP expression (62-64). In our study, expression of DSPP was weak before Day 7, but the amount increased by Day 10, and after Day 14 DSPP was strongly expressed (Fig. 7A). As a regulator of DSPP, the three miRNAs existed
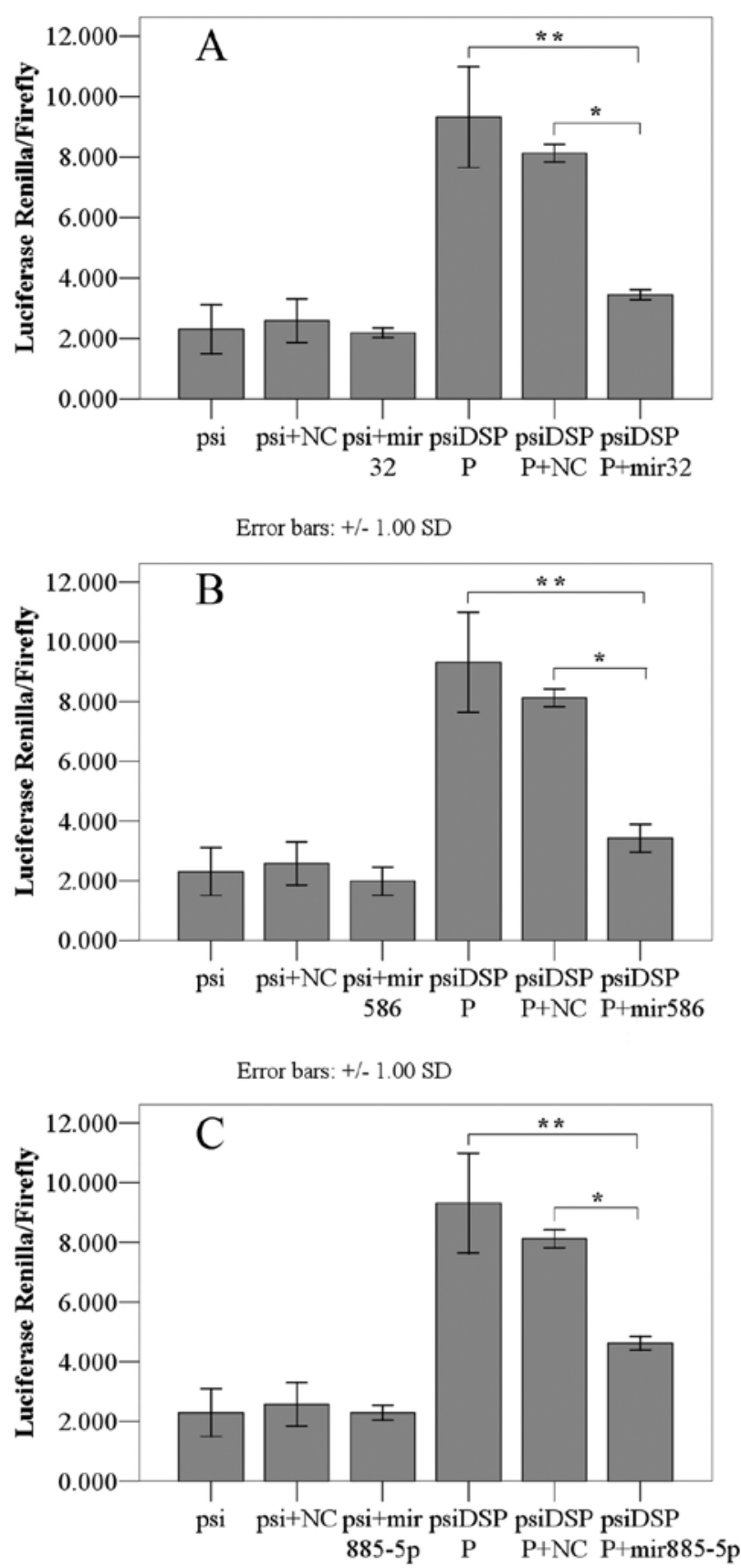

Error bars: +/- $1.00 \mathrm{SD}$

Figure 4. Luciferase reporter assay of (A) mir32; (B) mir586 and (C) mir885$5 \mathrm{p}$. Statistical analyses indicates that in the group of cells transfected with the luciferase vector alone, the binding ability of miRNAs were similar to $\mathrm{NC}$ (negative control) and blank psi luciferase vector alone. (A) In the group of cells transfected with DSPP 3'UTR vector, mir32 was able to reduce luciferase activity significantly compared with the blank $\left(\mathrm{P}=0.022\right.$, $\left.{ }^{*} \mathrm{P}<0.05\right)$ and $\mathrm{NC}\left(\mathrm{P}=0.003,{ }^{* *} \mathrm{P}<0.01\right)$ controls. (B) In the group of cells transfected with the DSPP 3'UTR vector, mir586 was able to reduce luciferase activity significantly compared with the blank $\left(\mathrm{P}=0.0179,{ }^{*} \mathrm{P}<0.05\right)$ and $\mathrm{NC}\left(\mathrm{P}=0.0072,{ }^{* *} \mathrm{P}<0.01\right)$ controls. (C) In the group of cells transfected with the DSPP 3'UTR vector, mir885-5p was able to reduce luciferase activity significantly compared with the blank $\left(\mathrm{P}=0.048,{ }^{*} \mathrm{P}<0.05\right)$ and $\mathrm{NC}\left(\mathrm{P}=0.0010,{ }^{* *} \mathrm{P}<0.01\right)$ controls.

in undifferentiated and differentiated dental pulp cells. The expression on Day 14 was significantly decreased, especially mir586, when DSPP was at its peak. After Day 14, expression levels of the three miRNAs were lower than undifferentiated 

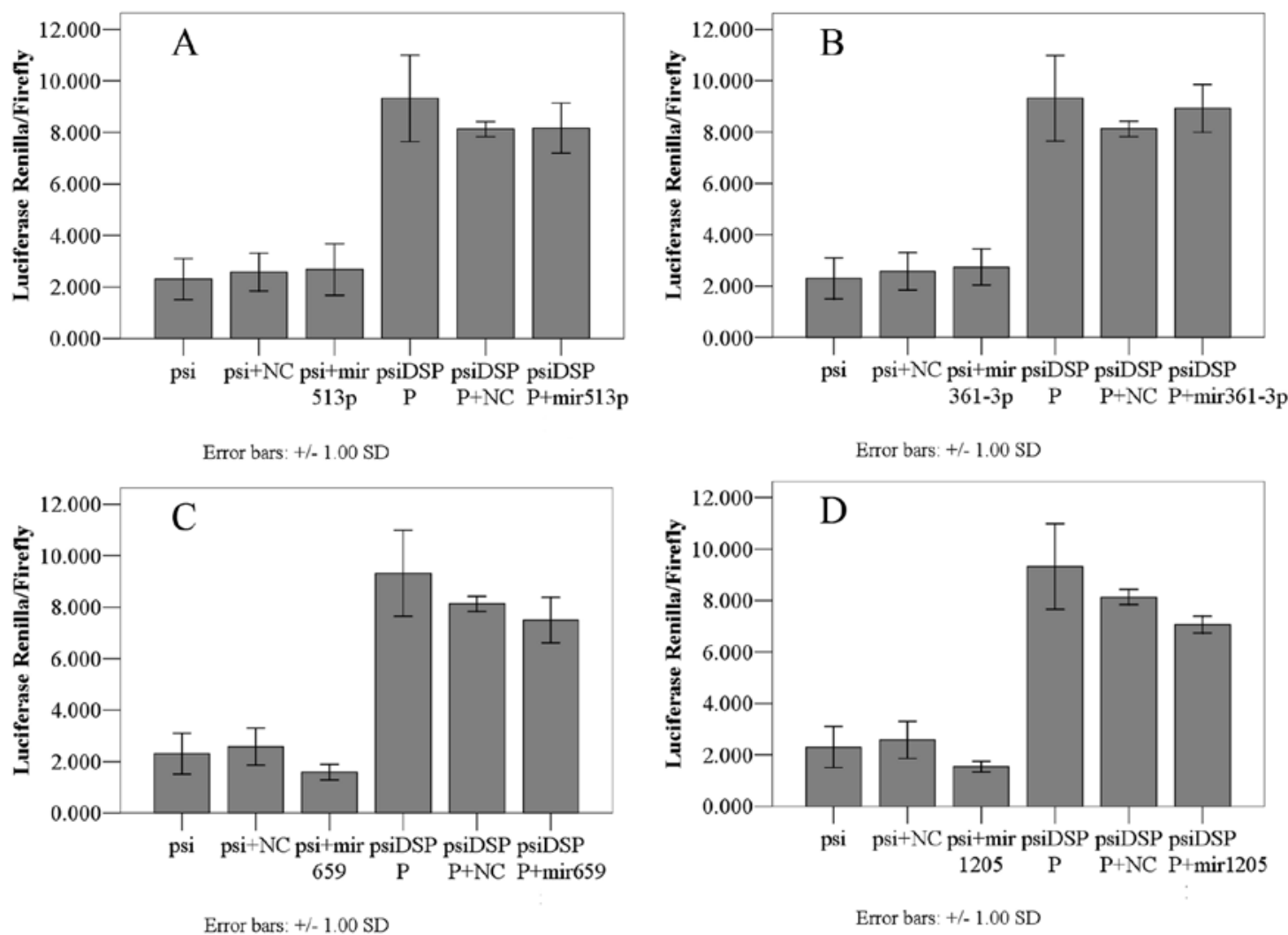

Error bars: $+/-1.00 \mathrm{SD}$

Error bars: +/- $1.00 \mathrm{SD}$

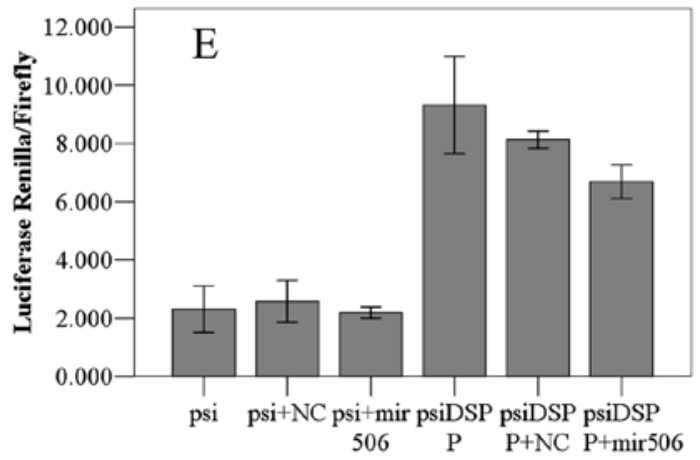

Error bars: $+/-1.00 \mathrm{SD}$

Figure 5. Luciferase reporter assay for (A) mir513p; (B) mir361-3p; (C) mir659; (D) mir1205 and (E) mir506. Statistical analyses indicates that in the group of cells transfected with the luciferase vector alone, the binding ability of miRNAs was almost the same to the NC and blank controls. In the group of cells transfected with the DSPP 3'UTR vector, these miRNAs were not able to reduce luciferase activity significantly compared with the blank and NC controls.

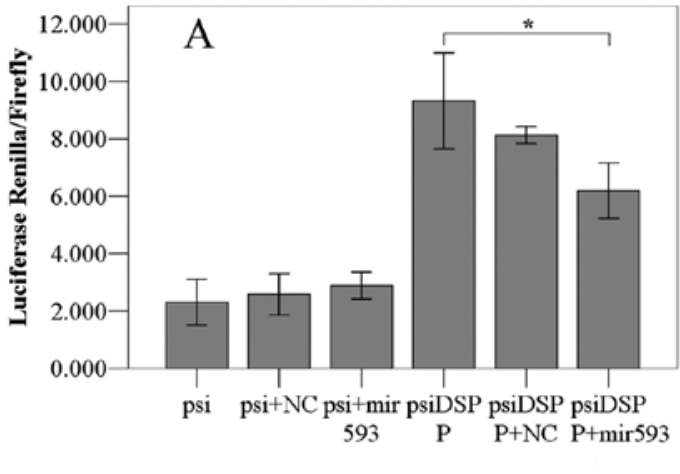

Error bars: $+/=1.00 \mathrm{SD}$

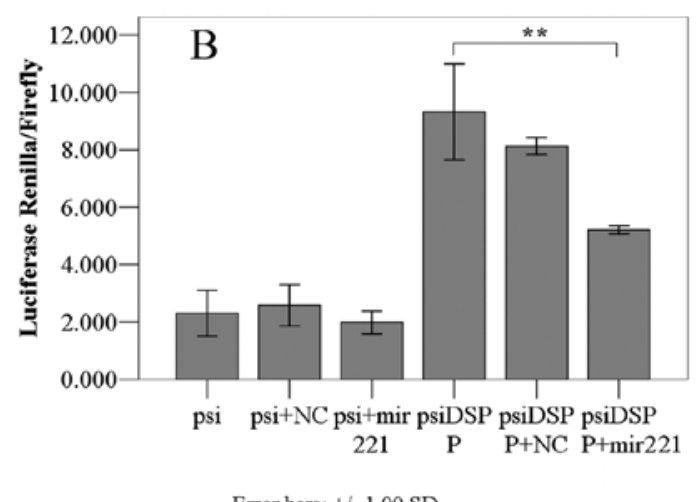

Error bars: $+/-1.00 \mathrm{SD}$

Figure 6. Statistical analyses indicates that in the group of cells transfected with the luciferase vector alone, the binding ability of miRNAs were similar to the NC and blank controls. (A) In the group of cells transfected with the DSPP 3'UTR vector, mir593 was able to inhibit luciferase activity significantly compared with the NC $\left(\mathrm{P}=0.0486,{ }^{\prime} \mathrm{P}<0.05\right)$, but not with the blank. (B) In the group of cells transfected with the DSPP $3^{\prime} \mathrm{UTR}$ vector, mir221 was able to inhibit luciferase activity significantly compared with $\mathrm{NC}\left(\mathrm{P}=0.001,{ }^{* * *} \mathrm{P}<0.01\right)$, but not with the blank. 

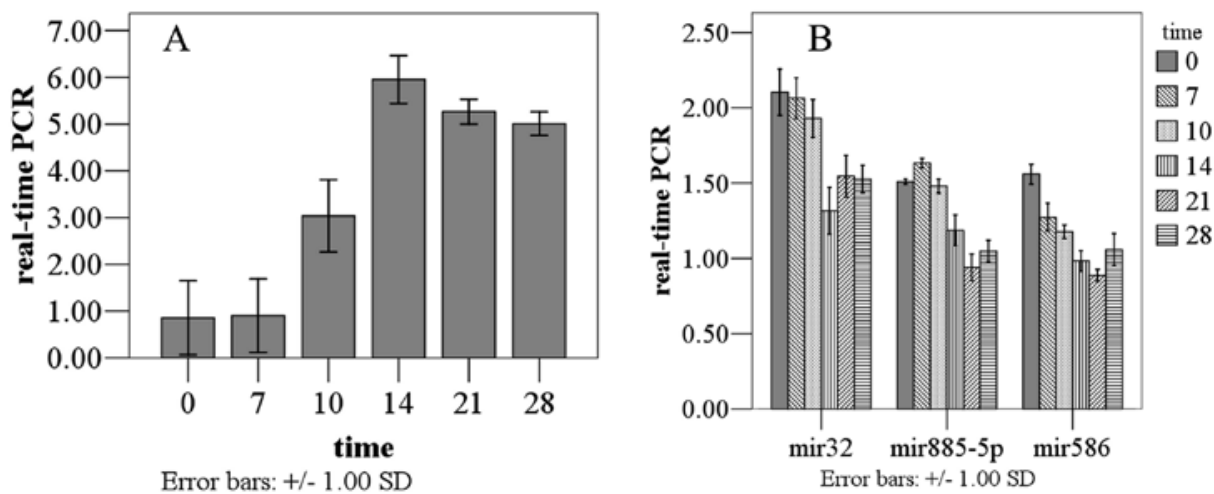

Figure 7. qRT-PCR for (A) DSPP and (B) mir32, mir885-5p and mir586 at different times during mineralized culture of dental pulp cells. (A) Expression of DSPP was weak before Day 7, but was increased on Days 10 and 14. (B) The 3 miRNAs existed in undifferentiated and differentiated dental pulp cells. After Day 10, the expression of especially mir885-5p and mir586 was less than that on Day 0.

cells, but the relative expression of mir32 was reduced less than that of the other two. On Days 21 and 28, mir885-5p and mir586 both maintained low expression levels (Fig. 7B).

\section{Discussion}

miRNAs are small non-coding RNA molecules that regulate gene expression post-transcriptionally during development and tissue homeostasis. It has been shown that some miRNAs can control gene expression during mesenchymal differentiation (65). There is a large variety of cells in the dental pulp, including the dentin-adherent odontoblasts, fibroblasts, undifferentiated mesenchymal cells, and structural cells of blood vessels. Transcription factor, growth factor and the matrix environment could induce dental pulp cells, including undifferentiated mesenchymal cells, into odontoblast-like cells. This is the first study to explore the effects of miRNAs in dental pulp cell differentiation, especially on the marker gene DSPP. Our goal was not only to identify miRNAs that regulate the specific gene DSPP of dental pulp cells, but also to predict a subset of miRNAs that could be regulated during differentiation of dental pulp cells and result in abnormal dentin formation.

These observations suggest that the expression of the specific gene DSPP during dentinogenesis is governed not only by differentially expressed transcription factors and growth factors $(61,66-70)$, but also by differentially expressed miRNAs.

In the present study, we predicted miRNAs targeting DSPP using computational analyses. Small differences in the implementation of the rules of these algorithms such as miRanda and TargetScan contributed to the discrepancies among their predicted targets. Despite the lack of sufficient numbers of experimentally verified targets for accurate assessment of the overall sensitivity and specificity of the predictions obtained by these algorithms, recent reports indicate that a large class of miRNA targets can be confidently predicted $(22,46)$.

To validate these predicted miRNAs, the dual luciferase reporter assay is a quick, sensitive and direct method. Combined with qRT-PCR, it confirmed that mir885-5p, mir586 and mir32 were expressed during differentiation of dental pulp cells to odontoblast-like cells. The results showed that on Day 14 the expression level of DSPP was highest, and the three miRNAs were lowest. When the level of DSPP was low, miRNAs were relatively high, which demonstrated that there was some link between DSPP and miRNAs. The nonlinear relationship between miRNAs and DSPP allows speculation that the three miRNAs regulate other target genes during differentiation. It has been reported that miRNAs show diverse regulatory mechanisms with mRNAs, including down-regulatory modules, up-regulatory modules, and mixed-regulatory modules. Even some of the earlier studies advocated that the miRNAs targeting the same gene together with a transcription factor might be regulated by the same transcription factor (71). Therefore, there is a complex regulatory network between miRNA and mRNA. The exact function of these miRNAs remains to be determined, but we believe this study provides a basis for further investigation of their function in dentinogenesis.

\section{Acknowledgements}

This study was supported by the Science and Technology Planning Project of Guangdong Province, China (2008B011300018-8), the Doctoral Foundation for The New Scholars of Ministry of Education of China (20094433120004) and the Natural Science Foundation of Guangdong Province, China (8451051501000371).

\section{References}

1. MacDougall M, Simmons D, Luan X, Nydegger J, Feng J and $\mathrm{Gu}$ TT: Dentin phosphoprotein and dentin sialoprotein are cleavage products expressed from a single transcript coded by a gene on human chromosome 4 . Dentin phosphoprotein DNA sequence determination. J Biol Chem 272: 835-842, 1997.

2. D'Souza RN, Cavender A, Sunavala G, et al: Gene expression patterns of murine dentin matrix protein 1 (Dmp1) and dentin sialophosphoprotein (DSPP) suggest distinct developmental functions in vivo. J Bone Miner Res 12: 2040-2049, 1997.

3. Qin C, Brunn JC, Cadena E, et al: The expression of dentin sialoprotein gene in bone. J Dent Res 81: 392-394, 2002.

4. Fisher LW, Jain A, Tayback M and Fedarko NS: Small integrin binding ligand $\mathrm{N}$-linked glycoprotein gene family expression in different cancers. Clin Cancer Res 10: 8501-8511, 2004.

5. Chaplet M, Waltregny D, Detry C, Fisher LW, Castronovo V and Bellahcene A: Expression of dentin sialophosphoprotein in human prostate cancer and its correlation with tumor aggressiveness. Int J Cancer 118: 850-856, 2006.

6. Ogbureke KU and Fisher LW: Renal expression of SIBLING proteins and their partner matrix metalloproteinases (MMPs). Kidney Int 68: 155-166, 2005. 
7. Ogbureke KU and Fisher LW: Expression of SIBLINGs and their partner MMPs in salivary glands. J Dent Res 83: 664-670, 2004.

8. Begue-Kirn C, Ruch JV, Ridall AL and Butler WT: Comparative analysis of mouse DSP and DPP expression in odntoblasts, preameloblasts, and experimentally induced odontoblast-like cells. Eur J Oral Sci 106 (Suppl 1): S254-S259, 1998.

9. Butler WT and Ritchie $\mathrm{H}$ : The nature and functional significance of dentin extracellular matrix proteins. Int J Dev Biol 39: 169-179, 1995.

10. Ritchie H, Bery JE, Somerman MJ, et al: Dentin sialoprotein (DSP) transcripts: developmentally-sustained expression in odontoblasts and transient expression in pre-ameloblasts. Eur J Oral Sci 105: 405- 413, 1997.

11. Xiao S, Yu C, Chou X, et al: Dentinogenesis imperfecta 1 with or without progressive hearing loss is associated with distinct mutations in DSPP. Nat Genet 27: 201-204, 2001.

12. Zhang X, Zhao J, Li C, et al: DSPP mutation in dentinogenesis imperfecta Shields type II. Nat Genet 27: 151-152, 2001

13. Rajpar MH, Koch MJ, Davies RM, Mellody KT, Kielty CM and Dixon MJ: Mutation of the signal peptide region of the bicistronic gene DSPP affects translocation to the endoplasmic reticulum and results in defective dentine biomineralization. Hum Mol Genet 11: 2559-2565, 2002.

14. Kim JW, Nam SH, Jang KT, et al: A novel splice acceptor mutation in the DSPP gene causing dentinogenesis imperfecta type II. Hum Genet 115: 248-254, 2004

15. Kim JW, Hu JC, Lee JI, et al: Mutational hot spot in the DSPP gene causing dentinogenesis imperfecta type II. Hum Genet 116 : 186-191, 2005 .

16. Malmgren B, Lindskog S, Elgadi A and Norgren S: Clinical, histopathologic, and genetic investigation in two large families with dentinogenesis imperfecta type II. Hum Genet 114: 491-498, 2004.

17. Holappa H, Nieminen P, Tolva L, Lukinmaa PL and Alaluusua S: Splicing site mutations in dentin sialophosphoprotein causing dentinogenesis imperfecta type II. Eur J Oral Sci 114: 381-384, 2006.

18. Zhang X, Chen L, Liu J, et al: A novel DSPP mutation is associated with type II dentinogenesis imperfecta in a Chinese family. BMC Med Genet 8: 52, 2007

19. Kida M, Tsutsumi T, Shindoh M, Ikeda $\mathrm{H}$ and Ariga T: De novo mutation in the DSPP gene associated with dentinogenesis imperfecta type II in a Japanese family. Eur J Oral Sci 117: 691-694, 2009.

20. Bai H, Agula H, Wu Q, et al: A novel DSPP mutation causes dentinogenesis imperfecta type II in a large Mongolian family. BMC Med Genet 11: 23, 2010.

21. Dong J, Gu T, Jeffords L and MacDougall M: Dentin phosphoprotein compound mutation in dentin sialophosphoprotein causes dentinogenesis imperfecta type III. Am J Med Genet A 132: 305-309, 2005

22. Sreenath T, Thyagarajan T, Hall B, et al: Dentin sialophosphoprotein knockout mouse teeth display widened predentin zone and develop defective dentin mineralization similar to human dentinogenesis imperfecta type III. J Biol Chem 278: 24874-24880, 2003.

23. Heikinheimo K: Stage-specific expression of decapentaplegic-Vgrelated genes 2,4, and 6 (bone morphogenetic proteins 2, 4, and 6 ) during human tooth morphogenesis. J Dent Res 73: 590-597, 1994

24. Chen Y, Bei M, Woo I, Satokata I and Maas R: Msx1 controls inductive signaling in mammalian tooth morphogenesis. Development 122: 3035-3044, 1996.

25. Tucker AS, Al Khamis A and Sharpe PT: Interactions between Bmp-4 and Msx-1 act to restrict gene expression to odontogenic mesenchyme. Dev Dyn 212: 533-539, 1998.

26. D'Souza RN, Aberg T, Gaikwad J, Cavender A, Owen M, Karsenty $\mathrm{G}$ and Thesleff I: Cbfal is required for epithelialmesenchymal interactions regulating tooth development in mice. Development 126: 2911-2920, 1999.

27. Laurikkala J, Mikkola M, Mustonen T, et al: TNF signaling via the ligand-receptor pair ectodysplasin and edar controls the function of epithelial signaling centers and is regulated by Wnt and activin during tooth organogenesis. Dev Biol 229: 443-455, 2001.

28. Payne TL, Skobe Z and Yelick PC: Regulation of tooth development by the novel type I TGFbeta family member receptor Alk8. J Dent Res 80: 1968-1973, 2001.

29. Xu X, Jeong L, Han J, Ito Y, Bringas P Jr and Chai Y: Developmental expression of Smad1-7 suggests critical function of TGF-beta/BMP signaling in regulating epithelial-mesenchymal interaction during tooth morphogenesis. Int J Dev Biol 47: 31-39, 2003.
30. Thesleff I: Epithelial-mesenchymal signalling regulating tooth morphogenesis. J Cell Sci 116: 1647-1648, 2003.

31. Xu X, Bringas P Jr, Soriano P and Chai Y: PDGFR-alpha signaling is critical for tooth cusp and palate morphogenesis. Dev Dyn 232: 75-84, 2005.

32. Sasaki T, Ito $\mathrm{Y}, \mathrm{Xu} X$, et al: LEF1 is a critical epithelial survival factor during tooth morphogenesis. Dev Biol 278: 130-143, 2005

33. Huang X, Xu X, Bringas P Jr, Hung YP and Chai Y: Smad4Shh-Nfic signaling cascade-mediated epithelial-mesenchymal interaction is crucial in regulating tooth root development. J Bone Miner Res 25: 1167-1178, 2010.

34. Ambros V: The functions of animal microRNAs. Nature 431: 350-355, 2004

35. Bartel DP: MicroRNAs: genomics, biogenesis, mechanism, and function. Cell 116: 281-297, 2004.

36. Bartel DP and Chen CZ: Micromanagers of gene expression: the potentially widespread influence of metazoan microRNAs. Nat Rev Genet 5: 396-400, 2004

37. Lim LP, Glasner ME, Yekta S, Burge CB and Bartel DP: Vertebrate microRNA genes. Science 299: 1540, 2003.

38. Lagos-Quintana M, Rauhut R, Lendeckel W and Tuschl T: Identification of novel genes coding for small expressed RNAs. Science 294: 853-858, 2001

39. Du T and Zamore PD: microPrimer: the biogenesis and function of microRNA. Development 132: 4645-4652, 2005.

40. Place RF, Li LC, Pookot D, Noonan EJ and Dahiya R: MicroRNA-373 induces expression of genes with complementary promoter sequences. Proc Natl Acad Sci USA 105: 1608-1613, 2008

41. Tay Y, Zhang J, Thomson AM, Lim B and Rigoutsos I: MicroRNAs to Nanog, Oct 4 and Sox 2 coding regions modulate embryonic stem cell differentiation. Nature 455: 1124-1128, 2008

42. Tsukamoto Y, Fukutani S, Shin-Ike T, Kubota T, Sato S, Suzuki Y and Mori M: Mineralized nodule formation by cultures of human dental pulp-derived fibroblasts. Arch Oral Biol 37: 1045-1055, 1992.

43. About I, Bottero MJ, de Denato P, Camps J, Franquin JC and Mitsiadis TA: Human dentin production in vitro. Exp Cell Res 258: $33-41,2000$

44. John B, Enright AJ, Aravin A, Tuschl T, Sander C and Marks DS: Human MicroRNA targets. PLoS Biol 2: e363, 2004.

45. John B, Sander C and Marks DS: Prediction of human microRNA targets. Methods Mol Biol 342: 101-113, 2006.

46. Griffiths-Jones S, Saini HK, van Dongen S and Enright AJ: miRBase: tools for microRNA genomics. Nucleic Acids Res 36 D154-D158, 2008.

47. Lewis BP, Burge CB and Bartel DP: Conserved seed pairing, often flanked by adenosines, indicates that thousands of human genes are microRNA targets. Cell 120: 15-20, 2005.

48. Chen C, Ridzon DA, Broomer AJ, et al: Real-time quantification of microRNAs by stem-loop RT-PCR. Nucleic Acids Res 33: e179, 2005.

49. Fiedler SD, Carletti MZ and Christenson LK: Quantitative RT-PCR methods for mature microRNA expression analysis. Methods Mol Biol 630: 49-64, 2010.

50. Chen C, Tan R, Wong L, Fekete R and Halsey J: Quantitation of microRNAs by real-time RT-qPCR. Methods Mol Biol 687: 113-134, 2011.

51. Gronthos S, Mankani M, Brahim J, Robey PG and Shi S: Postnatal human dental pulp stem cells (DPSCs) in vitro and in vivo. Proc Natl Acad Sci USA 97: 13625-13630, 2000.

52. Miura M, Gronthos S, Zhao M, Lu B, Fisher LW and Robey PG: SHED: stem cells from human exfoliated deciduous teeth. Proc Natl Acad Sci USA 100: 5807-5812, 2003.

53. Alliot-Licht B, Bluteau G, Magne D, Lopez-Cazaux S, Lieubeau B, Daculsi G and Guicheux J: Dexamethasone stimulates differentiation of odontoblast-like cells in human dental pulp cultures. Cell Tissue Res 321: 391-400, 2005.

54. Walsh S, Jordan GR, Jefferiss C, Stewart K and Beresford JN: High concentrations of dexamethasone suppress the proliferation but not the differentiation or further maturation of human osteoblast precursors in vitro: relevance to glucocorticoid-induced osteoporosis. Rheumatology (Oxford) 40: 74-83, 2001.

55. Pei W, Yoshimine $\mathrm{Y}$ and Heersche JN: Identification of dexamethasone-dependent osteoprogenitors in cell populations derived from adult human female bone. Calcif Tissue Int 72: 124-134, 2003.

56. Kasperk C, Schneider U, Sommer U, Niethard F and Ziegler R Differential effects of glucocorticoids on human osteoblastic cell metabolism in vitro. Calcif Tissue Int 57: 120-126, 1995. 
57. Kasugai S, Shibata S, Suzuki S, Susami T and Ogura H: Characterization of a system of mineralized-tissue formation by rat dental pulp cells in culture. Arch Oral Biol 38: 769-777, 1993.

58. Kuo MY, Lan WH, Lin SK, Tsai KS and Hahn LJ: Collagen gene expression in human dental pulp cell cultures. Arch Oral Biol 37: 945-952, 1992

59. Couble ML, Farges JC, Bleicher F, Perrat-Mabillon B, Boudeulle $\mathrm{M}$ and Magloire $\mathrm{H}$ : Odontoblast differentiation of human dental pulp cells in explants cultures. Calcif Tissue Int 66: 129-138, 2000 .

60. Ritchie HH, Park H, Liu J, Bervoets TJ and Bronckers AL: Effects of dexamethasone, vitamin A and vitamin D3 on DSP-PP mRNA expression in rat tooth organ culture. Biochim Biophys Acta 1679: 263-271, 2004

61. Chen S, Gu TT, Sreenath T, Kulkarni AB, Karsenty G and MacDougall M: Spatial expression of Cbfa1/Runx2 isoforms in teeth and characterization of binding sites in the DSPP gene. Connect Tissue Res 43: 338-344, 2002.

62. Kitagawa M, Ueda H, Iizuka S, et al: Immortalization and characterization of human dental pulp cells with odontoblastic differentiation. Arch Oral Biol 52: 727-731, 2007.

63. Nomiyama K, Kitamura C, Tsujisawa T, Nagayoshi $M$, Morotomi T, Terashita M and Nishihara T: Effects of lipopolysaccharide on newly established rat dental pulp-derived cell line with odontoblastic properties. J Endod 33: 1187-1191, 2007.
64. Pääkkönen V, Vuoristo JT, Salo T and Tjäderhane L: Comparative gene expression profile analysis between native human odontoblasts and pulp tissue. Int Endod J 41: 117-127, 2008.

65. Goff LA, Boucher S, Ricupero CL, et al: Differentiating human multipotent mesenchymal stromal cells regulate microRNAs: prediction of microRNA regulation by PDGF during osteogenesis. Exp Hematol 36: 1354-1369, 2008.

66. Lian JB, Stein JL, Stein GS, et al: Runx2/Cbfa1 functions: diverse regulation of gene transcription by chromatin remodeling and co-regulatory protein interactions. Connect Tissue Res 44 (Suppl 1): 141-148, 2003

67. Chen S, Rani S, Wu Y, et al: Differential regulation of dentin sialophosphoprotein expression by Runx2 during odontoblast cytodifferentiation. J Biol Chem 280: 29717-29727, 2005.

68. Yamashiro T, Zheng L, Shitaku Y, et al: Wnt10a regulates dentin sialophosphoprotein mRNA expression and possibly links odontoblast differentiation and tooth morphogenesis. Differentiation 75: 452-462, 2007.

69. Chen S, Gluhak-Heinrich J, Wang YH, et al: Runx2, osx, and dspp in tooth development. J Dent Res 88: 904-909, 2009.

70. Casagrande L, Demarco FF, Zhang Z, Araujo FB, Shi S and Nor JE: Dentin-derived BMP-2 and odontoblast differentiation. J Dent Res 89: 603-608, 2010.

71. Hornstein E and Shomron N: Canalization of development by microRNA. Nat Genet 38 (Suppl): S20-S24, 2006. 